\title{
儿童新型冠状病毒肺炎的防治
}

\author{
申 强 ${ }^{1}$ 赵 靖 ${ }^{1}$ 王荣福 ${ }^{1,2 *}$
}

1. 北京大学国际医院核医学科, 北京 102206

2. 北京大学第一医院核医学科, 北京 100034

摘 要: 随着新型冠状病毒肺炎疫情发展, 儿童新发新冠肺炎病例数增加, 为了明确诊断, 全国多地区开展新冠 肺炎病原检测, 发现儿童感染例数有明显的增加。在疫情期间, 需要保护儿童生命安全, 消除儿童恐惧感。因此, 需 要加强对儿童新型冠状病毒感染的肺炎的认识，防止误诊并早期诊治。

关键词：新冠肺炎；疫情防控；儿童

\section{Prevention and Control of COVID-19 of Children}

\author{
Qiang Shen ${ }^{1}$, Jing Zhao ${ }^{1}$, Rong-Fu Wang ${ }^{1,2 \star}$
}

1. Department of Nuclear Medicine, Peking University International Hospital, Beijing 102206, China

2. Department of Nuclear Medicine, Peking University First Hospital, Beijing 100034, China

\begin{abstract}
With the initial development of novel coronavirus epidemic situation, the number of new cases of coronal pneumonia among children has increased. In order to make clear the diagnosis and carry out pathogen detection of novel crown pneumonia in many regions of the country, it is found that the number of children infected has increased significantly. During the epidemic, children's lives need to be protected and their fears need to be eliminated. Therefore, we need to enhance the understanding of novel coronavirus pneumonia of children to prevent misdiagnosis, and conduct early diagnosis and treatment.
\end{abstract}

Keywords: COVID-19; plague control and prevention; children

一、前言

2019年底至今，一场突如其来的传染性肺炎疫情逐渐席卷全球。在这类最早出现于中国湖北省武汉市的肺炎感染 患者的上皮细胞中, 研究人员很快发现并证实为一种新型的冠状病毒, 2020年2月11日, 国际病毒分类委员会将其命 名为SARS-CoV-2 (severe acute respiratory syndrome coronavirus 2$)^{[1]}$, WHO将该病毒引起的肺炎正式命名为COVID-19 ( coronavirus disease 2019) ${ }^{[2]}$, 即新型冠状病毒肺炎 ( 以下简称为新冠肺炎)。全体人群对COVID-19普遍易感, 尤其 是儿童, 由于其免疫系统尚未发育成熟, 使得儿童亦成为该疾病的易感人群之一。目前来看, 虽然儿童新冠肺炎的感 染率和发病人数远远低于成人, 但随着疫情的不断进展, 儿童确诊例数也在日益增加 ${ }^{[3]}$ 。目前报道的患儿多为家庭聚 集性病例, 首发症状多为发热和/或呼吸道症状。相对于成人, 儿童病例症状较轻、病程较短、预后较好 ${ }^{[4-5]}$, 少数患 儿病情较重, 发病后症状无改善或加重, 出现低氧血症、ARDS、休克等症状。儿童感染病例数随着疫情进人高峰阶 段以及病原学检测工作的普遍展开，报告日益增多 ${ }^{[6]}$ ，但目前尚无儿童确诊病例死亡报道。

目前研究已经证实, COVID-19的传播途径主要是呼吸道飞沫和密切接触传播, 而新冠病毒感染患者是其主要传 染源, 但无症状感染者也不排除具有传染性, 可能成为其另一传染来源 ${ }^{[7]}$ 。除此之外, 新型冠状病毒在一些患者的粪

*通讯作者: 王荣福, 1955年9月, 男, 汉族, 福建南平人, 现任北京大学第一医院教授, 主任医师, 博士生导 师; 北京大学国际医院核医学科主任，医学和药学博士。研究方向：临床与分子核医学、放射性药学。

基金项目: 北京市科技计划项目（首都健康保障培育研究专项课题：Z181100001618017）；北京大学医一信交叉 项目（BMU2018MI009，BMU2018MI010）; 国家重大科学仪器设备开发专项（2011YQ03011409）; 十二五国家支 撑项目基金（2014BAA03B03） 
便及尿液中也被分离得到, 说明存在经粪口或气溶胶传播的可能, 因为因此需特别注意粪便及尿液的处理, 以免其长 时间密闭污染环境，造成高浓度的气溶胶，从而引起病毒的进一步传播和扩散 ${ }^{[7]}$ 。

新冠肺炎感染的主要临床表现是发热、乏力或者干咳等 ${ }^{[7,8,9]}$, 也可表现为非典型症状或者无临床症状 ${ }^{[10]}$ 。儿童病 例的临床症状相对成人普遍较轻, 而且一些儿童及新生儿病例患者仅仅表现为呼吸急促或精神状态差, 或者出现消化 道症状如呕吐、腹泻等，临床表现并不典型。

在已报道的儿童新冠肺炎确诊病例中, 发病年龄最小者 1 个月, 最大者 17 岁 ${ }^{[10]}$ 。患儿多由家长陪同来院就诊, 且 多为家庭聚集性发病, 因此需认真准确的对患儿及家长进行门急诊就诊前流行病调查。就诊前常规测量体温, 并填 写 “门急诊流行病调查表” ，此举是高效快速的篮查手段。通过门急诊人口、预检处、候诊区及诊室医生逐步深人问 诊等层层篎查, 疑似患儿的漏诊率可最大程度地降低。这种多层笁查流程的施行, 可有效提高儿童或婴幼儿疑似病例 的检出率，以便医务人员及时发现并控制传染源，尽量减少交叉感染，从而实现早发现、早隔离、早诊断、早治疗的 “四早”防控原则，使患儿受益。

\section{二、需严格执行分诊}

(一) 医院门急诊

承担着作为笁查新型冠状病毒肺炎的重要防控责任, 是最容易被感染的高危场所, 因此需做好阻断传播和扩散的 每一道关口。患儿及家长需提前在预就诊医院预约挂号, 采取非接触挂号方式, 如微信、医院APP、电话等平台, 并 如实填报相关信息。

\section{（二）来院就诊时}

患儿及家长务必佩戴好口罩 ( 儿童使用口罩时, 应在家长的帮助下佩戴儿童专用口罩), 由指定出人口进出门急 诊, 门急诊出人口施行单向通道。患儿及家长应到达就诊医院后, 应在分诊区进行初次的信息篎查, 并接受医务人员 对其进行体温测量。根据国家卫生健康委办公厅发布的新型冠状病毒肺炎诊疗方案（试行第七版）, 一旦发现就诊患 儿及其陪同人员具有如下临床症状和流行病学史中的任意一条, 均应立刻启动院内新型传染病紧急处理流程, 以排除 并及时管理疑似病例。

1. 临床症状

发热 (体温 $\geqslant 37.3^{\circ} \mathrm{C}$ ) 和/或呼吸道症状。

2. 流行病学史

( 1 ) 发病前14天内有疫区及周边地区的旅行史或居住史。

(2) 发病前14天内与新型冠状病毒感染者（核酸检测阳性者）有接触史。

( 3 ) 发病前 14 天内曾接触过来自疫区及周边地区，或来自有病例报告地区的发热或有呼吸道症状的患者。

( 4 ) 聚集性发病 ${ }^{[7]}$ 。

（三）候诊区二次篮查

患儿及陪同人员进人候诊区前, 需再次进行体温测量, 并向就诊科室的分诊护士出示就诊前的初次流行病学调查 表，待分诊人员完成预检，并且就诊患儿及陪同家属信息篎查符合规定者，方可进人候诊区等待就诊。

（四）诊室三级耖查

患儿就诊期间, 严格执行 “一人一陪同一诊室” 制度, 在进人诊室后, 由陪同家属出示就诊前流行病调查表由就 诊医生再次审核，诊室医生进一步篮查可疑症状及流行病学史，以防疑似病例漏诊。

\section{三、门急诊冠状病毒肺炎院内处理流程}

分诊护士在预检处或就诊医生在诊室内一旦篮查出疑似患儿, 应立即将患儿和家长就地隔离。经主诊医师及院内 专家会诊后, 如仍考虑疑似病例, 需将患儿及家长经指定通道带到指定地点, 在确保转运安全前提下立即将疑似病例 转运至定点医院。预检处与患者密切接触者或诊室医生应一并隔离。

\section{四、严格执行个人防护措施}

患儿及家长外出必须佩戴口罩、勤洗手, 家庭成员应掌握基本且必要的传染病防护知识及卫生消毒技能, 协助并 监管幼儿做好自我防护; 对于疑似新型冠状病毒肺炎的患儿及家长应监督其正确佩戴N95口罩或医用外科口罩, 在相 关医务人员引导下, 听从专业人员安排, 到指定区域内进行医学观察和处理, 未经相关负责人员许可, 不得私自离开 
指定区域。

\section{五、病例分类及处理原则}

对于发热的患儿, 快速病原学可对季节性病毒 (如腺病毒、流感病毒等) 进行快速笁查。之后, 感染及发热门 诊需再次根据患儿的症状、流行病学史、胸部 CT影像学表现等决定对患儿采取居家观察、收人普通病房或是收人 隔离病房治疗等不同措施。胸部CT检查有助于新型冠状病毒肺炎的及时诊断, 但儿童轻症病例胸部CT可无明显异 常。流行病学史是儿童新型冠状病毒肺炎感染病例早期发现和诊断的重要依据。疑似病例需进行新型冠状病毒核酸 检测。

(一) 居家观察患儿

医护人员应对患儿家属进行宣教, 指导居家隔离患儿及家属做好个人防护, 告知观察期间的注意事项及随访要 求, 并远程指导。患儿尽可能单间居住, 保持房间通风, 保证充足的营养, 维持水、电解质及酸碱平衡, 密切检测体 温及其他临床症状。

(二) 儿童COVID-19疑似病例

COVID-19疑似患儿病例, 在详细登记信息后, 应立即由专人引导且经专用通道进入隔离病房观察治疗。时间间 隔需 $\geqslant 24 \mathrm{~h}$ 后再次行新型冠状病毒核酸检测以明确有无感染。疑似病例应严格执行隔离措施, 单人单间隔离, 婴幼 儿、新生儿及年龄较小的儿童可与指定人员陪同隔离, 或者在确保安全转运前提下, 使用专用车辆将疑似病例转运至 定点医院进一步排查和确认, 及时进行新冠病毒的病原学检测。

连续两次新型冠状病毒核酸检测为阴性 (采样时间 $\geqslant 24 \mathrm{~h}$ ) , 且第三次新型冠状病毒特异性抗体 IgM和 IgG仍为 阴性可排除疑似病例诊断。

排除疑似可能性后，情况允许尽可能采用非接触式随访（电话或微信视频等）。

(三) 儿童COVID-19确诊病例

根据患儿及其密切接触者的COVID-19流行病学史和COVID-19主要症状和体征评估, 可分为四种临床分型: 轻 型、普通型、重型和危重型 ${ }^{[7]}$ 。第七版新型冠状病毒肺炎诊疗方案中新增了儿童患者重型标准，有如下五条：

1. 在无发热和哭闹的情况下, 出现呼吸频率增快;

2. 平静呼吸状态下，指氧饱和度不应超过 $92 \%$;

3. 出现缺氧症状: 需要辅助呼吸（呻吟、鼻翼煽动、三凹征）, 发绀, 间歇性呼吸暂停;

4. 有意识障碍表现: 嗜睡、惊厥;

5. 拒食或喂养困难，有脱水征。

普通型患儿需住院治疗，重型及危重型患儿需人住儿童重症医学病房。

对于重型及危重型患儿, 在一般对症治疗的基础上, 要积极采取综合措施以维持各重要脏器功能, 包括氧疗、机 械通气、充分液体复苏等呼吸、循环支持, 及早识别休克，避免重要脏器功能损伤，防治并发症。

目前尚未研制出治疗COVID-19的特效药, 主要以预防以及对症支持疗法为主 ${ }^{[11]}$ 。对于儿童新冠肺炎患者, 应遵 循儿科疾病的诊治常规, 积极防治并发症, 预防继发感染。发热时首选物理降温, 高热时再使用退烧药物, 强调及时 化痰祛痰。有学者认为, 对于儿童新型冠状肺炎, 一旦出现呼吸困难和低氧血症（血氧饱和度 $\leqslant 95 \%$ ) 时就应开始 给予有效的有氧治疗, 根据病情及时调整氧流量及给氧方式。一旦病情进展, 出现呼吸困难, 应首选无创机械通气更 为有利。如仍无法纠正缺氧或反复的呼吸暂停, 则应行有创机械通气, 必要时可考虑使用ECMO。儿童重型及危重型 病例可酷情考虑给予静脉滴注免疫球蛋白 ${ }^{[7]}$ 或其他治疗方案, 但应避免常规使用激素及盲目或不恰当的使用抗生素。

确诊新冠肺炎的患儿必须满足以下条件: 日常体温必须恢复连续正常 3 天以上, 原有的呼吸道症状明显改善, 复查的 胸部CT呈现肺部炎症明显吸收，连续2次新型冠状病毒核酸检测均阴性（2次之间至少间隔 $24 \mathrm{~h}$ ) 方可解除隔离出院。

基于当前儿童病例的诊治情况及相关文献报道 ${ }^{[12,13]}$, 与成人相比, 多数儿童患者预后良好, 病程较短, 肺部病灶 较局限, 多在 1 至 2 周内即可恢复。马慧静等对 22 例新冠肺炎儿童感染患者进行了总结研究, 分析其原因可能有以下两 点 ${ }^{[13]}$ : 儿童, 尤其是新生儿及婴幼儿发病, 多因与家庭成员密切接触而表现为家庭聚集性, 但感染时已多为第二代或 第三代感染, 病毒的毒力已经大大减弱; 儿童自身免疫系统尚未发育完善, 病毒诱发的免疫应答水平较低, 机体 “炎 症因子风暴”相对较轻有关。虽然儿童感染病例多为轻症，但家长如果察觉到异常情况，应及时就医。 


\section{六、结束语}

COVID-19为新型的重大传染性疾病, 随着全球儿童病例数及儿童相关病例的增加, 对各医院儿童病历资料的收 集与分析、笁查等有一定的帮助。儿童患者相对较少, 可能是因为家长对儿童采取较好的保护措施, 也可能与儿童免 疫力比较低，形成抗体和交叉免疫有关。因此，疫情流行期间，儿童应尽量避免外出，不到人员密集和密闭的场所。 此外, 家长应积极的进行亲子活动，包括安全、有益的适合儿童的体育活动，关注重视儿童心理健康，但避免亲密行 为；保证儿童良好的情绪、规律的作息，培养良好的行为习惯；做好手卫生，注意通风，保证充足的睡眠，合理均衡 的营养，达到吃动平衡。

\section{参考文献:}

[1]GORBALENYA AE,BAKER SC,BARIC RS,et al. Severe acute respiratory syndrome-related coronavirus The species and its viruses-a statement of the Coronavirus Study Group[J].bio Rxiv, 2020:2020-2022.DOI:10.1101/2020.02.07.937862.

[2]World Health Organization. WHO Director-General's remarks at the media briefing on 2019-nCoV on 11 February 2020 [ EB/OL ] . (2020/2/18) [ 2020-02-21 ] .https://www. who. int/dg/speeches/detail/who-directorgeneral-s-remarks-at-themedia-briefing-on-2019-ncov-on-11-february-2020.

[3]应艳琴,温宇,赵瑾珠.2019-nCoV病毒感染流行期间儿童分级防控建议[J].中国儿童保健杂志, 2020:1-11.

[4]重庆医科大学附属儿童医院新型冠状病毒感染诊治专家组,许红梅,罗征秀.儿童新型冠状病毒感染诊疗方案(重庆 医科大学附属儿童医院试行第二版)[J]. 儿科药学杂志, 2020:1-8.

[5]杨斌斌,朱月玲.儿童与成人新型冠状病毒肺炎(COVID-19)流行病学与治疗的现状分析 [J].西安交通大学学报(䀢 学版), 2020:1-10.

[6]中华医学会儿科学分会, 中华儿科杂志编辑委员会. 儿童2019新型冠状病毒感染的诊断与防治建议(试行第一版) [EB/OL]. [2020-02-05]. https://www. dxy. cn/bbs/newweb/pc/post/42754376.

[7]中华人民共和国国家卫生健康委员会. 新型冠状病毒肺炎诊疗方案(试行第七版)[EB/OL].(2020-03-03)[2020-0304].http://www.nhc.gov.cn/yzygj/s7653p/202003/46c9294a7dfe4cef80dc7f5912eb1989.shtml.

[8]CHEN N,ZHOU M,DONG X.Epidemiological and clinical characteristics of 99 cases of 2019 novel coronavirus pneumonia in Wuhan,China:a descriptive study [J].Lancet, 2020,395(10223):507-513.

[9]WANG D,HU B,HU C,et al. Clinical characteristics of 138 hospitalized patients with 2019 novel coronavirusinfectedpneumonia in Wuhan,China[J]. JAMA,2020.DOI:10.1001/jama.2020.1585.

[10]湖北省医学会儿科学分会,武汉医学会儿科学分会,湖北省儿科医疗质量控制中心.湖北省儿童新型冠状病毒感 染诊疗建议(试行第一版)[J].中国当代儿科杂志, 2020, 22(2):96-99.

[11]王琛,王旋.新型冠状病毒感染的流行、医院感染及心理预防[J].全科护理, 2020:1-2.

[12]姜毅,徐保平,金润铭.儿童新型冠状病毒感染诊断、治疗和预防专家共识(第一版)[J].中华实用儿科临床杂志, 2020,35(2):81-85.

[13]马慧静,邵剑波,王永姣.新型冠状病毒肺炎儿童高分辨率 CT 表现 [J/OL].中华放射学杂志, 2020,54(2020-0210).http://rs.yiigle.com/yufabiao/1180136.htm. doi:10.3760/cma.j.issn.1005-1201.2020.0002. MA Huijing, SHAO Jianbo, WANG Yongiiao, et al. High resolution CT features of novel coronavirus pneumonia in children [J]. Chin J Radiol, 2020, 54: doi:10.3760/cma.j.issn.1005-1201.2020.0002. 